\title{
Los regímenes internacionales de derechos humanos: la brecha entre compromiso y cumplimiento*
}

\section{Alejandro Anaya Muñoz**}

\section{RESUMEN}

A lo largo de las últimas siete décadas, se ha desarrollado un denso entramado de normas y órganos internacionales para la promoción y la protección de los derechos humanos en el mundo. Este andamiaje institucional conforma lo que la literatura de Relaciones Internacionales Ilama un "régimen internacional". ¿Qué tanto ha logrado el régimen internacional de derechos humanos su objetivo de promover y proteger los derechos en el mundo? ¿Qué tan bien equipado está para ello? ¿Cuáles son sus alcances y sus limitantes? Este artículo ofrece una primera aproximación a los argumentos y la evidencia empírica que la literatura de Relaciones Internacionales ha desarrollado al respecto. El artículo enfatiza la falta de "dientes" de los regimenes internacionales de derechos humanos y la brecha entre el compromiso y el cumplimiento de las normas internacionales en la materia y concluye con una reflexión sobre los retos de los regímenes internacionales para afrontar su limitado impacto sobre el comportamiento de los estados en la práctica.

PALABRAS CLAVE: Derechos humanos, régimen internacional, normas internacionales, órganos internacionales, compromiso y cumplimiento.

\begin{abstract}
During the past seven decades, the international community has developed a dense set of international organs and norms for the promotion and protection of human rights in the world. This institutional machinery conforms what the International Relations literature would call an "international regime." To what extent has the international human rights regime achieved its objective-the promotion and protection of human rights? How well equipped is it to do so? Which are its assets and its main limitations? This article offers a preliminary approach to the arguments and the empirical evidence that the International Relations literature has developed around this issue. The article underlines the regime's lack of "teeth" and the gap between commitment and compliance with international human rights norms and concludes with a discussion on the challenges faced by the regime to address its limited impact on state behaviour in practice.
\end{abstract}

KEY WORDS: human rights, international regime, international norms, international organs, commitment and compliance.

\footnotetext{
* Artículo recibido el 12 de enero de 2017 y aceptado el 27 de marzo de 2017.

** Profesor investigador, División de Estudios Internacionales, Centro de Investigación y Docencia Económicas (CIDE). (alejandro.anaya@cide.edu)
} 


\section{SUMARIO}

1. Introducción

2. Los órganos de los regímenes universal e interamericano de derechos humanos

3. Niveles de compromiso

4. Niveles de cumplimiento

5. Conclusiones

\section{Introducción}

A lo largo de las últimas siete décadas, se ha desarrollado a nivel internacional un denso entramado de normas y órganos internacionales para la promoción y la protección de los derechos humanos en el mundo. Este andamiaje institucional conforma lo que la literatura de las relaciones internacionales llama un "régimen internacional": un tipo de institución internacional integrado por principios, normas, reglas y procedimientos de toma de decisiones, establecido por los Estados para regular o moldear sus interacciones alrededor de un área temática específica de la esfera internacional. ${ }^{1}$

Los regímenes internacionales de derechos humanos son lo que en la literatura jurídica y en el mundo de los "practicantes" se conoce, sin demasiada precisión conceptual, como "los sistemas" de protección; como el "sistema" universal, el interamericano, el europeo o el africano. ¿Qué tanto los regímenes internacionales de derechos humanos han logrado promover y proteger dichos derechos? ¿Qué tan bien equipados están para ello? ¿Cuáles son sus alcances y limitantes? ¿En qué medida su existencia ha logrado promover y proteger los derechos humanos o, en otras palabras, qué tanto ha logrado influir en los niveles de vigencia de los derechos humanos en la práctica? Estas son las preguntas clave que la literatura de las relaciones internacionales se ha estado planteando sobre los derechos humanos durante al menos los últimos veinte años. Este artículo ofrece una primera aproximación a los argumentos teóricos y la evidencia empírica que se han desarrollado al respecto.

\footnotetext{
${ }^{1}$ Krasner, Stephen, International Regimes, Cambridge, Cornell University Press, 1983; Hasenclever, AndReAs, Mayer, PeTER y RITHBERger, VolKer, Theories of International Regimes, Cambridge, Cambridge University Press, 1997, pp. 8-22; Donnelly, JACK, "International Human Rights: A Regime Analysis", International Organization, vol. 40, núm. 3, 1986, pp. 599-605.

2 Sepúlveda, Magdalena, et al., Human Rights Reference Handbook, San José, Costa Rica, Universidad de la Paz, 2004; Martin, Claudia, Rodriguez-Pinzón, Diego y Guevara, José Antonio, Derecho Internacional de los Derechos Humanos, México, Universidad Iberoamericana - Washington College of Law - Fontamara, 2004; Steiner, Henry J., Alston, Philip y Goodman, Ryan, International Human Rights in Context. Law, Politics, Morals, Oxford, Oxford University Press, 2008.
} 
La primera sección del artículo describe "el corazón operativo" de los regímenes internacionales de derechos humanos: los órganos internacionales de promoción y defensa, particularmente de los regímenes universal e interamericano. ${ }^{3}$ En su segunda sección, se ofrece una aproximación descriptiva al grado de compromiso que los Estados han asumido con las normas de los regímenes internacionales de derechos humanos. En la tercera sección, el artículo desarrolla una evaluación preliminar sobre qué tanto los regímenes internacionales de derechos humanos han influido en el comportamiento de los Estados y, por tanto, en los niveles reales de vigencia de los derechos humanos en la práctica. De esta manera, se plantea un acercamiento empírico a la cuestión del compromiso con las normas del régimen internacional de derechos humanos. El artículo concluye con una breve reflexión sobre la brecha entre compromiso y cumplimiento y los retos de los regímenes internacionales de derechos humanos en la materia.

\section{Los órganos de los regímenes universal e interamericano de derechos humanos}

El régimen internacional de derechos humanos es aquel que, establecido por los Estados, emana de los principios de dignidad, igual valor e igualdad en derechos de la persona humana, así como de inalienabilidad, universalidad, interdependencia e indivisibilidad de los derechos humanos. El régimen, asimismo, se sustenta en un conjunto específico de normas positivas (establecidas en una amplia gama de instrumentos jurídicos internacionales). Cuenta para su funcionamiento u operación con un conjunto de órganos especializados de toma de decisiones e implementación de normas. Hablar del "régimen internacional de derechos humanos" (en singular), no obstante, sugiere la noción de un conjunto de principios, normas y órganos de toma de decisiones unificado. Sin embargo, la realidad empírica es amplia y diversa. Aun cuando los principios no varían y las normas en términos generales son similares, podemos hablar de la existencia de distintos regímenes de derechos humanos en la práctica.

Los instrumentos internacionales que contienen las normas de derechos humanos son numerosos, así como los órganos para la toma de decisiones y la implementación de normas. Es posible y de hecho necesario, descriptiva y analíticamente, agrupar los conjuntos normativos y los órganos internacionales de acuerdo con algún criterio de particularidad o afinidad. Por ejemplo, algunos

\footnotetext{
${ }^{3}$ El "régimen universal" es el que se ha desarrollado en el seno de la Organización de las Naciones Unidas (onu), mientras que el "régimen interamericano" lo ha hecho en el de la Organización de Estados Americanos (OEA).
} 
conjuntos de normas y órganos se relacionan de manera explícita con categorías amplias, pero particulares, de derechos, como los derechos civiles y políticos, por un lado, o los económicos, sociales y culturales, por el otro $;^{4} \mathrm{o}$ en derechos específicos, como la prohibición de la tortura o la desaparición forzada. Otros conjuntos de normas y órganos se pueden agrupar alrededor de los grupos específicos de sujetos a los cuales buscan proteger, como las mujeres, los niños y niñas, los trabajadores migratorios o las personas con discapacidad. En este sentido, podríamos hablar de distintos regímenes internacionales de derechos humanos, según los conjuntos de derechos o los grupos sociales en que se enfocan. Por mencionar únicamente un par de ejemplos, podríamos hablar del régimen internacional de derechos económicos, sociales y culturales o del régimen internacional de derechos del niño.

Sin embargo, la manera más común de desagregar el complejo régimen internacional de derechos humanos es alrededor de las distintas organizaciones internacionales en las cuales se insertan los distintos grupos concretos de normas y órganos existentes. Así, podemos hablar del régimen de la Organización de las Naciones Unidas (onU), o régimen universal; del régimen del Consejo de Europa o europeo; del régimen de la Organización de Estados Americanos (OEA) o interamericano, o del régimen de la Unión Africana (UA) o africano. ${ }^{5}$ Este criterio de clasificación o agrupación es el que se sigue en este trabajo por dos motivos. Por un lado, coincide con la práctica más común, tanto en otras disciplinas (como el derecho) como en el mundo de los "practicantes", en donde se suele hablar del "sistema" de derechos humanos universal, europeo, interamericano o africano. Por el otro, enfatiza el papel clave de las organizaciones internacionales (tanto la onU como distintas organizaciones regionales), no solamente en la promoción y defensa de los derechos humanos en el mundo, sino en el desarrollo normativo e institucional del sistema internacional; tema particularmente relevante para las relaciones internacionales. ${ }^{6}$ El análisis que se hace en este capítulo se centra solamente en los regímenes universal e interamericano.

\footnotetext{
${ }^{4}$ Esta categorización, no obstante, se encontraría un tanto en tensión con el principio de indivisbilidad.

${ }^{5}$ Los regímenes universal, europeo, interamericano y africano de derechos humanos son los que cuentan con un mayor grado de institucionalidad. Existen, no obstante, intentos más recientes e incipientes de desarrollo de un andamiaje de normas y órganos de derechos humanos en otros espacios regionales, como el Medio Oriente y el sudeste de Asia, o incluso en espacios culturales, como el mundo islámico.

${ }^{6}$ Evidentemente, no todas las corrientes teóricas de las relaciones internacionales estarian de acuerdo con la relevancia de dicha institucionalidad del sistema internacional y más en concreto en el área temática de los derechos humanos. Para una discusión, véase ANAYA MUÑOZ, ALEANDRo, Derechos humanos en y desde las Relaciones Internacionales, México, Centro de Investigación y Docencia Económicas, 2014, pp. 21-35.
} 
El régimen universal puede presentarse como uno compuesto por dos "sub-regímenes": el que surge de la Carta de la onu y el que se desprende de los tratados o convenciones internacionales adoptados en la materia. En otras palabras, por un lado, el régimen de la onu gira alrededor del Consejo de Derechos Humanos (Consejo DH), sucesor de la Comisión de Derechos Humanos (Comisión DH), por el otro, de los órganos de tratados u órganos convencionales: los comités que han surgido en virtud de los nueve tratados o convenciones principales de derechos humanos adoptadas en el marco de la onU. ${ }^{7}$

El Consejo DH -órgano que a partir de 2006 sustituyó a la Comisión DHse conforma por representantes de Gobiernos; lo cual, se dice, lo destina a la "politización": a dinámicas internas en las cuales tiende a prevalecer el interés nacional por encima de la promoción y protección de los derechos humanos. ${ }^{8}$ Ello no significa, necesariamente, que la situación real de vigencia o violación de los derechos humanos en un país no pueda ser un factor relevante en la toma de decisiones del Consejo DH. De hecho, la literatura ha demostrado, por ejemplo, que incluso la desprestigiada Comisión de Derechos Humano sí tomó en cuenta la situación de violaciones a los derechos humanos en la práctica al momento de adoptar resoluciones, particularmente tras el fin de la Guerra Fría. ${ }^{9}$

Parte fundamental del Consejo DH son sus "procedimientos especiales": relatores especiales, grupos de trabajo y "mandatos" similares para monitorear la situación de los derechos humanos en países concretos o sobre temas, derechos o grupos específicos. Estos procedimientos especiales son encomendados a expertos independientes, quienes actúan en su capacidad individual, sin representar a ningún Gobierno. El mismo es el caso para los órganos convencionales, los cuales también se conforman por un número variable de expertos independientes. En principio, entonces, parece ser que tanto los titulares de los procedimientos especiales como los órganos convencionales tienen mayor margen para actuar de manera objetiva o independiente que el Consejo DH, po-

\footnotetext{
${ }^{7}$ Estos órganos son: el Comité para la Eliminación de la Discriminación Racial; el Comité de Derechos Humanos; el Comité de Derechos Económicos, Sociales y Culturales; el Comité para la Eliminación de la Discriminación contra la Mujer; el Comité Contra la Tortura; el Subcomité para la Prevención de la Tortura; el Comité de los Derechos del Niño; el Comité de Derechos de los Trabajadores Migratorios; el Comité de los Derechos de las Personas con Discapacidad, y el Comité Contra las Desapariciones Forzadas.

${ }^{8}$ Lauren, PauL, "To Preserve and Build on its Achievements and to Redress its Shortcomings: The Journey from the Commission on Human Rights to the Human Rights Council", Human Rights Quarterly, vol. 29, núm. 2, 2007, pp. 307-345; anaYa muñoz, ALEandro, Derechos humanos en y desde las Relaciones Internacionales, México, Centro de Investigación y Docencia Económicas, 2014, pp. 68-74.

${ }^{9}$ Lebovic, James H. y Voeten, ERIK, "The Politics of Shame: The Condemnation of Country Human Rights Practices in the UNHCHR", International Studies Quarterly, vol. 50, núm. 4, 2006, pp. 861-888.
} 
tencialmente más vulnerable a dinámicas de politización. ${ }^{10}$ Esta intuición, no obstante, aún no ha sido rastreada empíricamente de manera explícita y sistemática por la literatura.

Las herramientas del Consejo DH para promover y proteger los derechos humanos en el mundo son la elaboración de propuestas de nuevos instrumentos normativos, el procedimiento de consideración de denuncias sobre situaciones de violaciones a los derechos humanos (heredero del procedimiento 1503 de la Comisión de Derechos Humanos), la adopción de resoluciones críticas y el establecimiento de los ya mencionados procedimientos especiales; los cuales, a su vez, realizan funciones de monitoreo (incluso mediante la realización de visitas in loco) y la elaboración de informes críticos al respecto. Más allá de estas funciones, con las cuales contaba también su antecesora, el Consejo DH tiene una nueva e importante herramienta para monitorear la situación de derechos humanos en todos los Estados miembro de la onU: el Examen Periódico Universal (EPU), mediante el cual se observa la situación de derechos humanos en todos los países del mundo, sin excepción, dentro de un esquema de evaluación periódica (en principio, cada cuatro años). De cualquier manera, el Consejo DH no tiene capacidad coercitiva: no puede tomar decisiones que impliquen medidas para obligar a los Estados a cumplir con sus recomendaciones. ${ }^{11}$

Los órganos convencionales de la onU, por su parte, tienen distintas funciones: revisión de informes periódicos de los Estados, consideración de comunicaciones individuales ${ }^{12}$ y (la mayoría de ellos) elaboración de investigaciones especiales. ${ }^{13} \mathrm{Al}$ igual que el Consejo DH y sus procedimientos especiales, los órganos de tratados solamente pueden emitir recomendaciones, las cuales no son vinculantes para los Estados. De nueva cuenta, estos órganos tampoco tienen capacidades coercitivas.

\footnotetext{
${ }^{10}$ anaYa muñoz, ALejandro, Derechos humanos en y desde las Relaciones Internacionales, México, Centro de Investigación y Docencia Económicas, 2014, pp. 75-78.

"Solamente el Consejo de Seguridad de la onu cuenta con capacidad coercitiva, actuando bajo el capítulo vII de la Carta de la Organización.

${ }^{12}$ Esta competencia no se activa "en automático" tras la ratificación del tratado en cuestión. Su reconocimiento es opcional para los Estados parte de cada tratado y se hace manifiesta mediante una declaración especial o la ratificación de un protocolo (lo cual varía en cada tratado). En el caso del Comité de Derechos de los Trabajadores Migratorios, su mecanismo de comunicaciones individuales aún no está vigente, dado que aún no cuenta con el número mínimo de notificaciones de aceptación de esta competencia por los Estados parte de la Convención correspondiente.

${ }^{13}$ Los órganos de tratados también pueden elaborar observaciones generales, mediante las cuales clarifican o desarrollan el contenido de los derechos enunciados en cada tratado o convención. Así mismo, la mayoría también pueden recibir comunicaciones estatales; es decir, una especie de denuncia entre Estados. Aunque este último mecanismo no ha sido utilizado en la práctica.
} 
Pasando al régimen interamericano, la Comisión Interamericana de Derechos Humanos (Comisión IDH) está conformada por siete expertos independientes que sirven en su capacidad individual; mientras que la Corte Interamericana de Derechos Humanos (Corte IDH) se conforma por el mismo número de jueces, quienes tampoco representan a los Gobiernos de sus países. ${ }^{14}$ Las principales funciones de la Comisión IDH son la elaboración de informes especiales (sobre la situación de un país, un derecho, tema o grupo específico) y la consideración de quejas o peticiones individuales. De nueva cuenta, la principal herramienta en ambos casos es la elaboración de recomendaciones, incluso en el caso del procedimiento de consideración de quejas individuales. Solamente la Corte IDH puede adoptar sentencias vinculantes. Los Estados tienen, en otras palabras, la obligación jurídica de implementar las medidas de reparación que la Corte IDH plantea en sus sentencias. Sin embargo, una vez más, ni la Comisión IDH ni la Corte IDH tienen capacidad coercitiva.

Podemos concluir que los órganos de los regímenes universal e interamericano de derechos humanos (al igual que los de los regímenes europeo y africano) "no tienen dientes”. Su principal herramienta es nombrar y señalar: mediante sus evaluaciones críticas y sus recomendaciones y sentencias, los órganos de los regímenes internacionales de derechos humanos evidencian de manera pública y legítima las brechas entre los compromisos adoptados por los Estados y su falta de cumplimiento en la práctica. ${ }^{15}$ Esta falta de capacidades coercitivas o para vincular de manera directa los derechos humanos con intereses materiales (en otras palabras, esa falta de "dientes") ha sido entendida como la principal causa de los bajos o decepcionantes niveles de cumplimiento.

\section{Niveles de compromiso}

A nivel agregado global, el nivel de ratificación de la mayoría de los tratados internacionales de derechos humanos es muy alto. Esto denota un elevado compromiso con las normas internacionales correspondientes. Como se puede observar en la tabla 1, con excepción de la Convención Internacional sobre la Protección de los Derechos de todos los Trabajadores Migratorios y de sus Familiares (adoptada desde 1990) y de la Convención Internacional para la

\footnotetext{
${ }^{14}$ anaYa muñoz, Alejandro, Derechos humanos en y desde las Relaciones Internacionales, México, Centro de Investigación y Docencia Económicas, 2014, pp. 79-84.

${ }^{15}$ ANAYA muÑOZ, ALejandro, Derechos humanos en y desde las Relaciones Internacionales, México, Centro de Investigación y Docencia Económicas, 2014, pp. 63-97.
} 
Protección de todas las Personas contra la Desaparición Forzada, el resto de los tratados han sido ratificados por muchos Estados.

Tabla 1. Principales tratados del régimen universal de derechos humanos y número de ratificaciones

\begin{tabular}{|l|c|c|}
\hline Tratado & $\begin{array}{c}\text { Año de } \\
\text { adopción }\end{array}$ & $\begin{array}{c}\text { Número de } \\
\text { ratificaciones }\end{array}$ \\
\hline $\begin{array}{l}\text { Convención Internacional sobre la Eliminación de } \\
\text { todas las Formas de Discriminación Racial }\end{array}$ & 1965 & 178 \\
\hline Pacto Internacional de Derechos Civiles y Políticos & 1966 & 169 \\
\hline $\begin{array}{l}\text { Pacto Internacional de Derechos Económicos, So- } \\
\text { ciales y Culturales }\end{array}$ & 1966 & 165 \\
\hline $\begin{array}{l}\text { Convención sobre la Eliminación de Todas las For- } \\
\text { mas de Discriminación contra la Mujer }\end{array}$ & 1979 & 189 \\
\hline $\begin{array}{l}\text { Convención contra la Tortura y Otros Tratos o Pe- } \\
\text { nas Crueles, Inhumanos o Degradantes }\end{array}$ & 1984 & 196 \\
\hline \begin{tabular}{l} 
Convención sobre los Derechos del Niño \\
\hline $\begin{array}{l}\text { Convención Internacional sobre la Protección de } \\
\text { los Derechos de todos los Trabajadores Migratorios } \\
\text { y de sus Familiares }\end{array}$
\end{tabular} & 1990 & 50 \\
\hline $\begin{array}{l}\text { Convención sobre los Derechos de las Personas con } \\
\text { Discapacidad }\end{array}$ & 2006 & 172 \\
\hline $\begin{array}{l}\text { Convención Internacional para la Protección de } \\
\text { todas las Personas contra la Desaparición Forzada }\end{array}$ & 2006 & 55 \\
\hline
\end{tabular}

Fuente: Elaboración propia con información de la Oficina del Alto Comisionado de las Naciones Unidas para los Derechos humanos.

Si rastreamos las tendencias a lo largo del tiempo, podemos identificar una evolución de los tratados de derechos humanos en el mundo: cada vez más países ratifican más tratados. ${ }^{16}$ Algunos tratados fueron ratificados por muchos

\footnotetext{
${ }^{16}$ LANDMAn, TodD, Protecting human rights: A comparative study, Washington DC, Georgetown University Press, 2005, pp. 62-66.
} 
Estados muy rápidamente (como la Convención sobre los Derechos del Niño o la Convención sobre la Eliminación de Todas las Formas de Discriminación contra la Mujer), mientras que otros han tenido un ritmo de ratificación mucho más lento (como la ya mencionada Convención sobre los Derechos de los Trabajadores Migratorios). Como quiera que sea, la tendencia global ha sido la de un constante aumento del número de Estados que han adquirido compromisos con las normas de los regímenes internacionales de derechos humanos.

Los países de América Latina son "ratificadores" ejemplares. Como se puede ver en la gráfica 1, con la excepción de Belice (que no ha asumido compromisos con los tratados del régimen interamericano), los Estados de la región han ratificado la mayoría de los diecisiete tratados más importantes de derechos humanos suscritos en el marco de la ONU y la OEA.

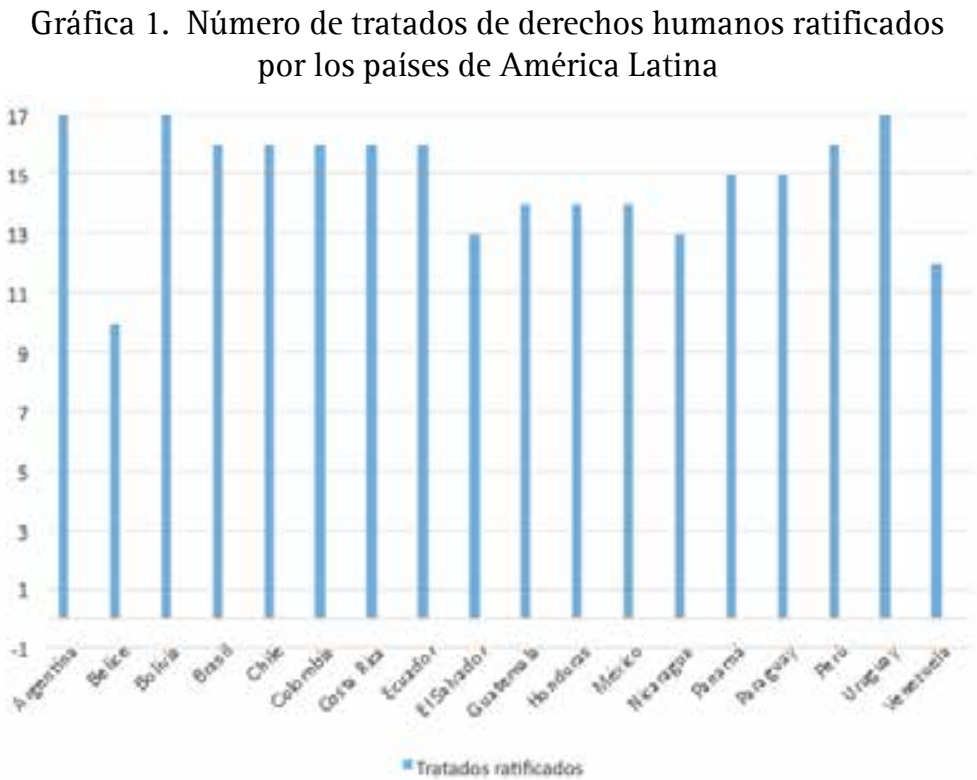

Fuente: elaboración propia con información de la Oficina del Alto Comisionado de las Naciones Unidas para los Derechos humanos y la Comisión Interamericana de Derechos Humanos. ${ }^{17}$ Nota: Venezuela ratificó la Convención Americana sobre Derechos Humanos en 1977; sin embargo, denunció dicho tratado el 10 de septiembre de 2012 (la denuncia entró en vigor un año después).

\footnotetext{
17 "Status of Ratifications: Interactive Dashboard", Oficina del Alto Comisionado de las Naciones Unidas para los Derechos Humanos. [Consulta: 17 de febrero, 2017]. Disponible en: http://indicators.ohchr.org/
} 
Desde una perspectiva histórica, observamos la ya mencionada tendencia incremental de ratificación de tratados. En 1980, solamente diez países habían ratificado al menos tres de los cinco tratados de derechos humanos adoptados hasta entonces. Por lo contrario, seis países habian ratificado solamente uno o ninguno de dichos tratados. Esta brecha se fue cerrando progresivamente a lo largo del tiempo: hacia 1990, trece países de la región habían ratificado al menos seis tratados; hacia el año 2000, quince países habían ratificado más de nueve tratados, y hacia el 2017, todos los países, con la ya señalada excepción de Belice, habían ratificado más de doce de los diecisiete tratados disponibles (véase gráfica 1). El compromiso con las normas de los regímenes universal e interamericano es evidente.

Otra manera de observar el compromiso con las normas internacionales de derechos humanos es la medida en que han aceptado (o no) la competencia o jurisdicción de los órganos internacionales para recibir y procesar quejas o comunicaciones individuales sobre casos concretos. La aceptación de esta competencia o jurisdicción (en el caso de la Corte IDH) es opcional para los Estados que han ratificado los tratados correspondientes. Si consideramos a esta facultad como más intrusiva de la soberanía nacional, en el sentido de que implica una determinación autoritativa y vinculante sobre la comisión de actos ilegales concretos, entonces los Estados que han aceptado esta facultad estarían demostrando un compromiso mayor con los regímenes internacionales de derechos humanos que los que no lo han hecho. La gráfica 2 muestra el grado en que los países de la región han reconocido la competencia de los órganos de tratados de la onu para recibir comunicaciones individuales. La variación entre países es importante; pero tomando en cuenta que son ocho los órganos que actualmente cuentan con esta competencia vigente, el nivel de compromiso que ello denota sigue siendo considerable a nivel regional.

Para concluir este análisis sobre el compromiso de los Estados con los órganos de los regímenes internacionales de derechos humanos, podemos explorar el reconocimiento de la jurisdicción de la Corte IDH para examinar casos

168 concretos de posible violación a los derechos humanos. Al igual que en el caso de los órganos convencionales de la onU, el reconocimiento de dicha jurisdicción es opcional para los Estados miembro de la oEA, e incluso para los que han ratificado la Convención Americana de Derechos Humanos (CADH). Todos los Estados latinoamericanos continentales (de nueva cuenta con la excepción de Belice) han reconocido la jurisdicción de la Corte IDH, si bien lo han hecho en momentos muy distintos: algunos desde principios de la década de los ochenta; otros, prácticamente veinte años después (véase tabla 2). Más allá de estas 
variaciones en el tiempo, la tabla 2 muestra que el compromiso de los Estados latinoamericanos con el régimen regional de derechos humanos es claro.

Gráfica 2. Reconocimiento de la competencia de los órganos convencionales de la onu para recibir comunicaciones individuales

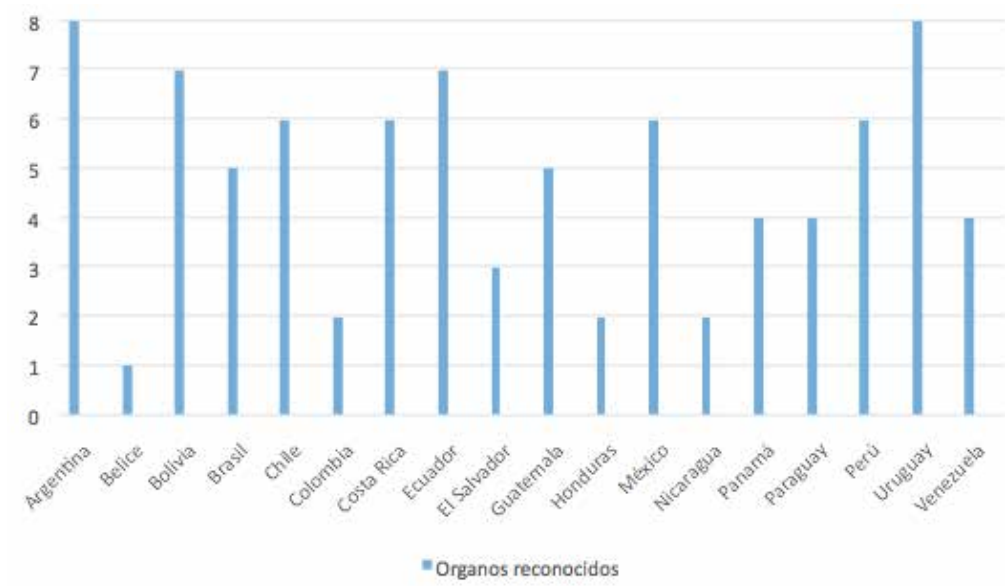

Elaboración propia con base en datos de la Oficina del Alto Comisionado de las Naciones Unidas para los Derechos Humanos. ${ }^{18}$

Tabla 2. Año de reconocimiento de la jurisdicción de la Corte IDH

\begin{tabular}{|l|c|}
\hline Argentina & 1984 \\
\hline Belice & \\
\hline Bolivia & 1993 \\
\hline Brasil & 1998 \\
\hline Chile & 1990 \\
\hline Colombia & 1985 \\
\hline Costa Rica & 1980 \\
\hline Ecuador & 1984 \\
\hline El Salvador & 1995 \\
\hline Guatemala & 1987 \\
\hline Honduras & 1981 \\
\hline México & 1998 \\
\hline
\end{tabular}

\footnotetext{
18 "Ratification Status Finder", Oficina del Alto Comisionado de las Naciones Unidas para los Derechos Humanos. [Consulta: 17 de febrero, 2017]. Disponible en: http://tbinternet.ohchr.org/_layouts/TreatyBodyExternal/Treaty.aspx?CountrylD $=7 \&$ Lang $=E N$
} 


\begin{tabular}{|l|l|}
\hline Nicaragua & 1991 \\
\hline Panamá & 1990 \\
\hline Paraguay & 1993 \\
\hline Perú & 1981 \\
\hline Uruguay & 1985 \\
\hline Venezuela & $1981^{*}$ \\
\hline
\end{tabular}

Fuente: elaboración propia con base en Comisión Interamericana de Derechos Humanos, "Documentos Básicos de Derechos Humanos en el Sistema Interamericano", Organización de Estados Americanos. [Consulta: 17 de febrero, 2017]. Disponible en: http://www.oas.org/es/cidh/mandato/documentos_basicos.asp *Nota: Venezuela denunció la caDH el 10 de septiembre de 2012 (la denuncia entró en vigor un año después).

En suma, podemos identificar una tendencia global, la cual se manifiesta claramente en América Latina, que muestra un progresivo compromiso con las normas y los órganos de los regímenes internacionales de derechos humanos. ${ }^{19}$ La pregunta obligada en este sentido es si este compromiso ha resultado en mayores niveles de cumplimiento.

\section{Niveles de cumplimiento}

Un problema importante en el desarrollo de una agenda de investigación sobre el cumplimiento de las normas internacionales en materia de derechos humanos es la falta de indicadores comprehensivos, comparables y confiables. ${ }^{20}$ Sin embargo, distintos esfuerzos de medición (sobre todo basados en la técnica de mediciones con base en estándares) ofrecen indicadores proxys que han permitido a la literatura de política comparada y relaciones internacionales acercarse a esta cuestión. Uno de dichos intentos ha sido el Cingranelli and Richards Human Rights Data Project (CIRI). ${ }^{21}$ Estos índices buscan reflejar, en una escala ordinal, los niveles agregados de respeto o violación de distintos derechos humanos en la práctica,

\footnotetext{
${ }^{19}$ Ciertamente, esta tendencia no es tan clara en otras regiones del planeta. Véase LANDMAN, Todd, Protecting human rights: A comparative study, Washington DC, Georgetown University Press, 2005, pp. 67-70.

${ }^{20}$ anAYA muñoz, Alejandro, Hacia una metodología para la medición del cumplimiento de los Derechos Humanos en México, México, Universidad Iberoamericana, 2008, pp. 9-26; ANAYA muÑoz, ALEjANDRo, "Indicadores de derechos humanos: tipos, métodos y bases de datos existentes", en Ariadna Estévez y Daniel Vázquez (coords.), Derechos Humanos y Transformación Política en Contextos de Violencia, México, Flacso - CISAN - UnAm, 2015, pp. 55-73.

${ }^{21}$ Cingranell, David, Richards, David \& Clay, Chad, Human Rights Data Project (ciri). [Consulta: 22 de febrero, 2017]. Disponible en: http://www.humanrightsdata.com

Desafortunadamente este proyecto de generación de indicadores ha sido cancelado recientemente. Los datos están disponibles hasta 2011. Otras alternativas son "The Political Terror Scale". [Consulta: 26 de febrero, 2017]. Disponible en: http://www.politicalterrorscale.org/ y los indices de libertades civiles y derechos políticos de "Freedom House". [Consulta: 26 de febrero, 2017]. Disponible en: https://freedomhouse.org/
} 
país por país y año con año (entre 1981 y 2011). Se basan en una bien diseñada y sistemática codificación de la información contenida en los informes anuales sobre los derechos humanos en el mundo, elaborados por Amnistía Internacional y el Departamento de Estado del gobierno de los Estados Unidos.

Utilizando los índices de derechos a la integridad física y de empoderamiento de CIRI, podemos acercarnos empíricamente a la situación de vigencia o cumplimiento de los derechos humanos en América Latina. En el índice de derechos a la integridad física se incluyen los indicadores de ejecuciones extrajudiciales, tortura, desaparición forzada y encarcelamiento político. Este índice se presenta en una escala de 0 a 8 , siendo 0 el mayor nivel de violación y 8 el de mayor respeto o vigencia. El índice de derechos de empoderamiento, por su parte, incluye indicadores sobre libertad de movimiento internacional, libertad de movimiento nacional, libertad de expresión, libertad de asamblea y asociación, derechos de los trabajadores, libre determinación electoral y libertad de religión. Este índice se presenta en una escala de 0 a 14 puntos: 0 implica el mayor grado de violación y 14 el mayor nivel de respeto o vigencia de los derechos en cuestión. ${ }^{22}$

En la gráfica 3 podemos observar que el promedio global del índice de derechos a la integridad física ha mejorado ligeramente en los 30 años observados; pasó de 3 a 5 puntos (8 es el nivel más alto de respeto). Por su parte, el promedio mundial de derechos de empoderamiento ha mejorado también, pasando de 5 a 8 puntos (14 es el mayor puntaje de la escala).

\footnotetext{
${ }^{22}$ La base de datos CIRI ofrece dos índices de derechos de empoderamiento: el "old empowerment rights index" y el "new empowerment rights index". En este artículo se utiliza el segundo de ellos.
} 
Gráfica 3. Derechos a la integridad física y derechos de empoderamiento.

Promedio global (1981-2011)

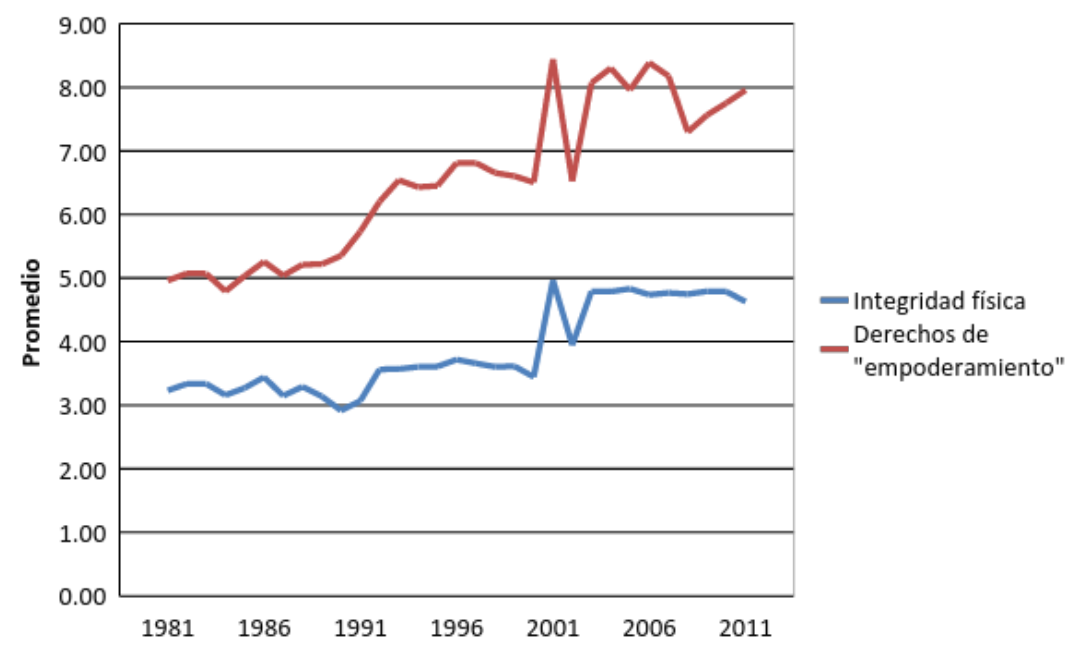

Elaboración propia con datos de Human Rights Data Cingranelli (n 21)

Nota: Datos solamente disponibles hasta 2011.

La gráfica 4 presenta el índice cIRI de derechos a la integridad física para los países de América Latina. Podemos ver, para esta región, una tendencia muy similar a la observada a nivel global. En la década de los ochenta, el promedio regional de este índice varió entre los 3 y los 4 puntos. La situación en esta década fue claramente mala en la región. Para la primera década del 2000, el promedio fluctuó entre los 4 y 5 puntos, acercándose a un punto medio en la escala. La gráfica 5 nos muestra que en el caso de los derechos de empoderamiento, el promedio regional se acerca mucho más a la parte superior de la escala. Sin embargo, también podemos observar que la situación prácticamente no cambió entre 1983 y el 2011.

Como quiera que sea, los promedios regionales para los derechos de empoderamiento se han mantenido constantemente por encima de los mundiales. Esto sugiere que América Latina ha sido una región donde, después de todo, desde una perspectiva comparada, se ha gozado de un nivel razonable de libertad de movimiento internacional, libertad de movimiento nacional, libertad de expresión, libertad de asamblea y asociación, derechos de los trabajadores, libre determinación electoral y libertad de religión. No podemos decir lo mismo, no obstante, con respecto a los derechos a la integridad física. 
Gráfica 4. Índice de derechos a la integridad física. América Latina (1981-2011)

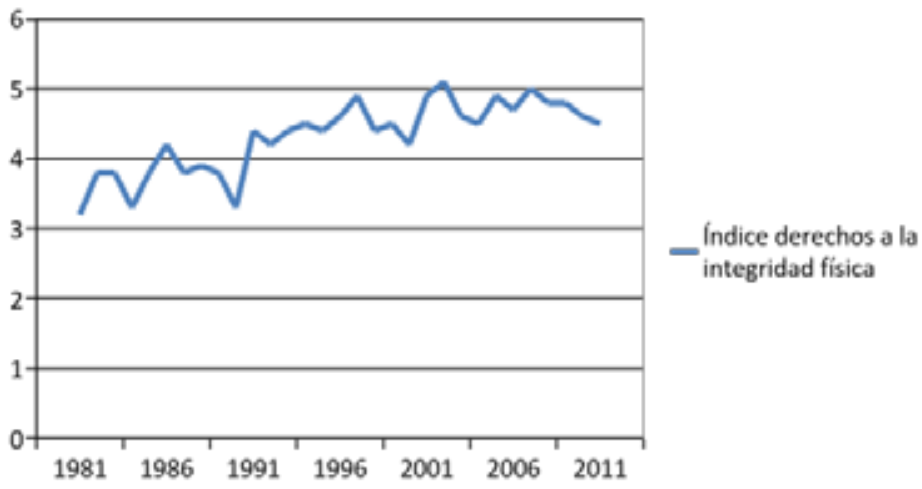

Elaboración propia con base en datos de www.humanrightsdata.com Nota: Datos solamente disponibles hasta 2011.

Gráfica 5. Índice de derechos de empoderamiento. América Latina (1981-2011)

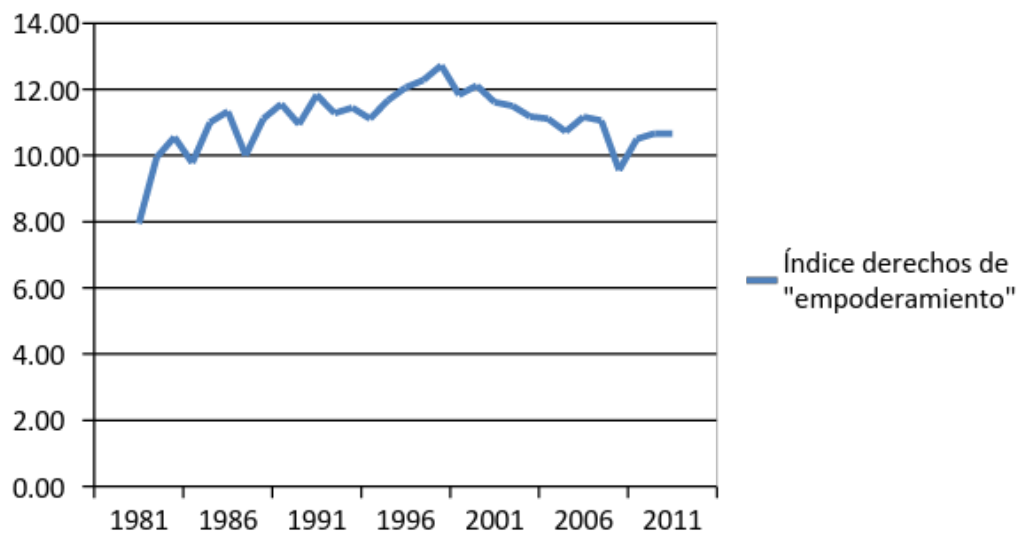

Elaboración propia con base en datos de www.humanrightsdata.com Nota: Datos solamente disponibles hasta 2011. 
En suma, la situación de vigencia de los derechos humanos en el mundo ha mejorado moderadamente durante los últimos 30 años (particularmente en lo que respecta a los derechos de empoderamiento). Es precisamente la etapa en que los regímenes internacionales de derechos humanos se han extendido y fortalecido. ${ }^{23}$ Sin embargo, esta mejoría ha sido más bien moderada o limitada (particularmente en derechos a la integridad física) y estamos lejos aún de alcanzar una situación satisfactoria. Más aún, la adopción de compromisos ha sido mucho más clara o contundente que los modestos cambios en los niveles de vigencia. ${ }^{24}$ Esto es lo que la literatura de relaciones internacionales ha identificado como una clara brecha entre "derechos en principio" y "derechos en la práctica" 25 o entre compromiso con y cumplimiento de las normas internacionales de derechos humanos. ${ }^{26}$

Utilizando técnicas estadísticas cada vez más sofisticadas, un creciente número de publicaciones han explorado la covariación entre la adopción de compromisos normativos (mediante la ratificación de tratados) y la situación de vigencia de los derechos a la integridad física en la práctica. ${ }^{27}$ Una primera ola de estudios, centrados en los derechos a la integridad física concluyó que la ratificación de tratados no solamente no estaba correlacionada con una mejoría en la vigencia de derechos, sino que, por lo contrario, los Estados violaban más derechos tras haber ratificado.

Una manera de explicar estos resultados fue que la ratificación era un ejercicio meramente instrumental por parte de los Estados, basado en un cálculo de costo-beneficio: los costos de ratificar son muy bajos (pues los regímenes internacionales de derechos humanos no tienen capacidad coercitiva), mientras que los beneficios en materia de legitimidad internacional son altos. Más aún, el aumento en legitimidad internacional podría dar a los países en cuestión mayor espacio para reprimir un poco más a sus disidentes internos. ${ }^{28}$

${ }^{23}$ Los indicadores de Freedom House reflejan una mejora aún más pronunciada. Sin embargo, los indicadores de la Political Terror Scale muestran una tendencia contraria (véase LANDMAN, Tod, Protecting human rights: A comparative study, Washington DC, Georgetown University Press, 2005, pp. 98-99).

${ }^{24}$ LANDMAN, TODD, Protecting human rights: A comparative study, Washington DC, Georgetown University Press, 2005, p. 28.

${ }^{25}$ Landman, Todd, Protecting human rights: A comparative study, Washington DC, Georgetown University Press, 2005. ${ }^{26}$ CÁRdenAS, SONIA, Conflict and Compliance. State Responses to International Human Rights Pressure, Philadelphia, University of Pennsylvania Press, 2007.

${ }^{27}$ No es claro por qué la inmensa mayoría de los autores no se han interesado por explorar estas relaciones en el caso de los derechos de empoderamiento.

${ }^{28}$ Hathaway, Oona, "Do human rights treaties make a difference?" The Yale Law Journal, vol. 11, núm. 8, 2002, pp. 1835-2042. 
Otros autores, no obstante, a pesar de que coincidieron en los nulos efectos de la ratificación, destacaron "la paradoja de las promesas vacías": a pesar de que en efecto la ratificación no estaba correlacionada directamente con un mejor comportamiento por parte de los Estados, sí aumentaba las herramientas normativas disponibles para los grupos de defensa de los derechos humanos. ${ }^{29}$ Otros estudios encontraron que la ratificación de tratados de derechos humanos sí tiene un impacto positivo sobre los niveles de vigencia; aunque el efecto es débil o moderado. ${ }^{30}$

Algunos más han encontrado que la ratificación de tratados ha tenido un impacto positivo en los niveles de vigencia de los derechos humanos bajo ciertas condiciones: en los países más democráticos y con una sociedad civil con vínculos con el exterior más fuerte. Por lo contrario, la ratificación no ha tenido efectos (o incluso ha tenido efectos negativos) en países autocráticos que no cuentan con una sociedad civil transnacionalizada fuerte. ${ }^{31}$ Finalmente, en el que bien puede ser el estudio más sofisticado y convincente en la materia, se ha concluido que la ratificación de tratados sí tiene una influencia positiva en los niveles de vigencia, particularmente en las democracias en transición o en flujo, las cuales cuentan con una sociedad civil que puede aprovechar las mejores oportunidades para el litigio y la movilización que propician la adopción de compromisos normativos internacionales por parte de sus Gobiernos. ${ }^{32}$

Otra manera de evaluar el cumplimiento de las normas internacionales de derechos humanos puede ser el observar la implementación de las recomendaciones o sentencias adoptadas por los órganos de los regímenes internacionales en la materia. Como se señaló en la sección 1 de este artículo, dichos órganos monitorean de manera crítica el comportamiento general de los Estados (su cumplimiento de las normas del régimen) y reciben casos concretos de presunta violación de los derechos humanos. El resultado de estas funciones de monitoreo y de revisión cuasi-jurisdiccional o jurisdiccional (en el caso de las cortes internacionales, como la interamericana o la europea) es la elaboración de recomendaciones o la adopción de sentencias. Mediante estas recomendaciones y las medidas de reparación elaboradas en las sentencias, los órganos de los regímenes internacionales plantean acciones concretas que los Estados deben

\footnotetext{
${ }^{29}$ Hafner-Burton, Emille \& Tsutsul, Kiyoteru, "Human Rights in a Globalizing World: The Paradox of Empty Promises", American Journal of Sociology, vol. 110, núm. 5, 2005, pp. 1373-1411.

${ }^{30}$ LANDMAn, Todd, Protecting human rights: A comparative study, Washington DC, Georgetown University Press, 2005.

${ }^{31}$ NeUMeYER, ERIK, "Do international human rights treaties improve respect for human rights?", Journal of Conflict Resolution, vol. 49, núm. 6, 2005, pp. 925-953.

32 Simmons, Betr, Mobilizing for Human Rights. International Law in Domestic Politics, Cambgridge, Cambridge University Press, 2009.
} 
implementar para cumplir con sus compromisos normativos internacionales, incluyendo la reparación del daño. ¿En qué medida los Estados implementan o ponen en la práctica estas recomendaciones o medidas de reparación?

La literatura que ha explorado este tipo de preguntas es menos abundante que la que explora el tema del impacto de la ratificación de tratados. Sin embargo, los estudios muestran que la implementación de las decisiones de los órganos de los regímenes internacionales de derechos humanos es muy limitada, tanto en el régimen universal como en los regionales. ${ }^{33}$ En los términos de una de esas investigaciones, "una crisis de implementación afecta actualmente a los órganos jurídicos regionales e internacionales encargados de la protección de los derechos humanos". ${ }^{34}$

En el caso de los órganos del régimen interamericano, el grado de implementación o cumplimiento de las recomendaciones de la Comisión IDH y las medidas de reparación establecidas en las sentencias de la Corte IDH es muy pobre. ${ }^{35}$ Uno de los estudios ya citados encuentra que hasta 2008 , solamente $12.5 \%$ de las recomendaciones de la Comisión IDH habían sido totalmente cumplidas; mientras que $69.5 \%$ de ellas habían sido cumplidas solamente de manera parcial y $18 \%$ no se había cumplió en ningún grado. Encuentra también que hasta 2010, la Corte IDH había adoptado un total de 126 sentencias, de las cuales solamente 6\% habían sido implementadas en su totalidad. El $61 \%$ de las sentencias se habían implementado de forma parcial, mientras que el resto no habían sido implementadas en ninguna medida. ${ }^{36}$ No resulta sorprendente, por otro lado, que los Estados cumplan más con cierto tipo de medidas; en concreto, el pago de indemnizaciones a las víctimas. Las medidas que se cumplen menos son las que implican reformar el marco jurídico del Estado o investigar y sancionar a los responsables de las violaciones a los derechos humanos. ${ }^{37}$

${ }^{33}$ Open SOCIETY InITIATIVE, From Judgement to Justice: Implementing International and Regional Human Rights Decisions, Nueva York, Open Society Foundations, 2010; BASCH, FernAndo, "La Efectividad del Sistema Interamericano de Protección de Derechos Humanos: Un Enfoque Cuantitativo sobre su Funcionamiento y sobre el Cumplimento de sus Decisiones", Sur Revista Internacional de Derechos Humanos, vol. 7, núm. 12, 2010, pp. 9-35.

${ }^{34}$ Open Society InITIATIVE, From Judgement to Justice: Implementing International and Regional Human Rights Decisions, Nueva York, Open Society Foundations, 2010, p. 11, traducción del autor.

${ }^{35}$ Para una reflexión sobre las diferencias entre "implementación" y "cumplimiento" véase Open SocIETY INITAATVE, From Judgement to Justice: Implementing International and Regional Human Rights Decisions, Nueva York, Open Society Foundations, 2010, p. 13.

${ }^{36}$ Open Society InITIATIVE, From Judgement to Justice: Implementing International and Regional Human Rights Decisions, Nueva York, Open Society Foundations, 2010.

${ }^{37}$ Open SocietY INITIATIVE, From Judgement to Justice: Implementing International and Regional Human Rights Decisions, Nueva York, Open Society Foundations, 2010, pp. 65-70. 
Otro estudio encuentra que la mitad de las decisiones o "remedios" recomendados u ordenados entre 2001 y 2011 por los órganos del régimen interamericano de derechos humanos no fueron cumplidas para nada; sólo 14\% han sido cumplidas de manera parcial y $36 \%$ han sido totalmente implementadas. De nueva cuenta, este estudio encuentra que las recomendaciones o "remedios" que más se cumplieron fueron las que pidieron una indemnización monetaria para las víctimas (con un grado de cumplimiento de 58\%) y las que solicitaron una reparación simbólica (cumplidas en 52\%). Por lo contrario, los "remedios" que menos se implementaron fueron los relacionados con investigar y sancionar: solamente $10 \%$ de ellos se cumplieron en su totalidad..$^{38}$

Desafortunadamente, no hay estudios sistemáticos sobre el nivel de implementación de las recomendaciones hechas en las decisiones sobre comunicaciones individuales adoptadas por los órganos convencionales de la onU. La tendencia, no obstante, es plantear que los órganos convencionales son "el eslabón más débil" del régimen de la onu y que por lo mismo los Estados suelen ignorar sus recomendaciones. ${ }^{39}$ Una manera (si bien imperfecta) de acercarse a un análisis sistemático al respecto es analizar los informes anuales de los órganos convencionales, en los cuales éstos hacen una valoración sobre las respuestas de los Estados a los dictámenes o decisiones sobre comunicaciones individuales.

Por ejemplo, tras la adopción de un informe de dictamen sobre una comunicación individual hecha por el Comité de Derechos Humanos (el órgano convencional del régimen universal que ha adoptado más decisiones sobre comunicaciones individuales), el Estado involucrado tiene que enviar al Comité una respuesta en la que establezca la manera en que pretende implementar las recomendaciones que se le hacen en la decisión. El Comité evalúa esta respuesta y determina si es satisfactoria, si considera que el Estado muestra la intención de implementar las recomendaciones o de ofrecer a las víctimas una reparación satisfactoria.

Hacia 2009, el Comité de Derechos Humanos había adoptado 546 dictámenes en los cuales encontró que en efecto se dieron violaciones a los derechos

\footnotetext{
${ }^{38}$ El estudio se basa en las recomendaciones hechas en informes de fondo y de soluciones amistosas de la Comisión DH y en las sentencias adoptadas por la Corte IDH entre 2001 y 2006 para el caso de los países que habian aceptado la jurisdicción de la Corte IDH. Véase BASCH, Fernando, "La Efectividad del Sistema Interamericano de Protección de Derechos Humanos: Un Enfoque Cuantitativo sobre su Funcionamiento y sobre el Cumplimento de sus Decisiones", Sur Revista Internacional de Derechos Humanos, vol. 7, núm. 12, 2010, pp. 11-12, 20 y 21; véase también Open SocietY InITIATIVE, From Judgement to Justice: Implementing International and Regional Human Rights Decisions, Nueva York, Open Society Foundations, 2010, p. 80.

${ }^{39}$ KRommendiJK, JASPER, "Finnish Exceptionalism at Play? The Effectiveness of the Recommendations of un Human Rights Treaty Bodies in Finland", Nordic Journal of Human Rights, vol. 32, núm. 1, 2014, p. 19.
} 
humanos contenidos en el Pacto Internacional de Derechos Civiles y Políticos (PIDCP). Solamente en $12 \%$ de ellos el Comité había recibido una respuesta de los Estados considerada como satisfactoria. En el resto, el Comité consideró que las respuestas fueron insatisfactorias o, peor aún, no recibió respuesta alguna. Si consideramos estos datos como un indicador (como se decía, imperfecto) del grado de implementación de las recomendaciones del Comité, encontraríamos entonces un nivel de cumplimiento claramente pobre. Peor aún, la implementación se habría deteriorado con el paso del tiempo, pues en su informe anual de 1999 el Comité de Derechos Humanos reportó que cerca de 30\% de las respuestas a sus informes de dictamen habían sido satisfactorias. ${ }^{40}$

\section{Conclusiones}

Este artículo recurre de manera explícita al concepto de "régimen internacional", característico de la disciplina de las relaciones internacionales, para realizar un acercamiento al conjunto de normas y órganos internacionales de derechos humanos existentes en la actualidad. Más allá de describir la composición y las funciones de los regímenes universal e interamericano de derechos humanos, el artículo explora, de manera preliminar, las cuestiones del compromiso y el cumplimiento de las normas del régimen por parte de los Estados, con un especial énfasis en América Latina. ¿Cuál ha sido el impacto o la influencia de las normas y órganos internacionales de derechos humanos en los niveles de vigencia de los mismos en la práctica? ¿Qué tanto los órganos de los regímenes internacionales han logrado que los Estados cumplan con las normas que se han comprometido? ¿En qué medida los Estados han implementado las decisiones de los órganos de los regímenes internacionales de derechos humanos?

El punto de partida para acercarnos a este análisis ha sido reconocer a los regímenes como instituciones internacionales "sin dientes": sin capacidad para forzar el cumplimiento de sus normas y de las decisiones de sus órganos. Por otro lado, se ha enfatizado la brecha entre compromiso y cumplimiento. Parece ser que la falta de cumplimiento tiene mucho que ver con la falta de capacidad coercitiva de los órganos de los regímenes. Su reto principal, entonces, es encontrar maneras para disminuir la brecha entre compromiso y cumplimiento, recurriendo a mecanismos no basados en la coerción ni en la generación directa de costos materiales para los Estados violadores de derechos humanos.

\footnotetext{
${ }^{40}$ Open SocietY InITIATIVE, From Judgement to Justice: Implementing International and Regional Human Rights Decisions, Nueva York, Open Society Foundations, 2010, pp. 26-27 y 117-121.
} 
La literatura ha empezado a enfocarse en las condiciones (principalmente internas) bajo las cuales es más probable que se dé el paso del compromiso al cumplimiento y en las que la presión normativa por parte de los órganos del régimen y otros actores internacionales no estatales tenga mayor impacto. ${ }^{41} \mathrm{Un}$ factor particular del cual la literatura de relaciones internacionales no se ha ocupado hasta el momento es cómo lograr mayores grados de implementación de las recomendaciones y sentencias concretas de los órganos internacionales.

La brecha entre el compromiso y el cumplimiento y los problemas de implementación de recomendaciones y sentencias, no obstante, no implican que los regímenes internacionales de derechos humanos hayan sido totalmente inconsecuentes, que su existencia no importe, o que no aporten nada a la vigencia o protección de los derechos humanos en el mundo. La situación de derechos humanos en el mundo ha mejorado a lo largo de las últimas décadas (si bien de manera moderada), y sería difícil plantear que los regímenes internacionales y los procesos transnacionales e internos que se dan alrededor de los derechos humanos no han tenido nada que ver. Los regímenes internacionales, en efecto, no tienen dientes. Pero gracias a ellos los actores (nacionales e internacionales) que se dedican a su promoción y defensa cuentan con más y mejores herramientas para perseguir sus objetivos y disminuir la brecha entre compromiso y cumplimiento. En este sentido, un factor clave para el desarrollo de la agenda de los derechos humanos en el mundo es la combinación virtuosa de un régimen internacional fuerte y una sociedad civil (nacional e internacional) movilizada.

\section{Bibliografía}

Anaya Muñoz, Alejandro, Hacia una metodología para la medición del cumplimiento de los Derechos Humanos en México, México, Universidad Iberoamericana, 2008.

Anaya Muñoz, Alejandro, Derechos humanos en y desde las Relaciones Internacionales, México, Centro de Investigación y Docencia Económicas, 2014.

Anaya Muñoz, Alejandro, "Indicadores de derechos humanos: tipos, métodos y bases de datos existentes”, en Ariadna Estévez y Daniel Vázquez (coords.), Derechos Humanos y Transformación Politica en Contextos de Violencia, México, Flacso - CISAN - UNAM, 2015.

Basch, Fernando, "La Efectividad del Sistema Interamericano de Protección de Derechos Humanos: Un Enfoque Cuantitativo sobre su Funcionamiento y sobre

\footnotetext{
${ }^{41}$ Risse, Thomas, Ropp, Stephen y Sikkink, Kathryn, The Persistent Power of Human Rights. From Commitment to Compliance, Cambridge, Cambridge University Press, Cambridge, 2013.
} 
el Cumplimento de sus Decisiones", Sur Revista Internacional de Derechos Humanos, vol. 7, núm. 12, 2010.

CÁrdenas, Sonia, Conflict and Compliance. State Responses to International Human Rights Pressure, Philadelphia, University of Pennsylvania Press, 2007.

Cingranelui, David, Richards, David \& Clay, Chad, Human Rights Data Project (ciri). [Consulta: 22 de febrero, 2017]. Disponible en: http://www.humanrightsdata.com

Donnelly, JACK, “International Human Rights: A Regime Analysis”, International Organization, vol. 40, núm. 3, 1986.

Hafner-Burton, Emilie \& Tsutsui, Kiyoteru, "Human Rights in a Globalizing World: The Paradox of Empty Promises", American Journal of Sociology, vol. 110, núm. 5, 2005.

Hasenclever, Andreas, Mayer, Peter y Rittberger, Volker, Theories of International Regimes, Cambridge, Cambridge University Press, 1997.

Hathanay, Oona, "Do human rights treaties make a difference?" The Yale Law Journal, vol. 11, núm. 8, 2002.

Krasner, Stephen, International Regimes, Cambridge, Cornell University Press, 1983. KrommendiJK, JASPeR, "Finnish Exceptionalism at Play? The Effectiveness of the Recommendations of un Human Rights Treaty Bodies in Finland", Nordic Journal of Human Rights, vol. 32, núm. 1, 2014.

Landman, Todd, Protecting human rights: A comparative study, Washington DC, Georgetown University Press, 2005.

Lauren, Paul, "To Preserve and Build on its Achievements and to Redress its Shortcomings': The Journey from the Commission on Human Rights to the $\mathrm{Hu}-$ man Rights Council”, Human Rights Quarterly, vol. 29, núm. 2, 2007.

Lebovic, James H. y Voeten, EriK, "The Politics of Shame: The Condemnation of Country Human Rights Practices in the UnHCHR”, International Studies Quarterly, vol. 50, núm. 4, 2006.

Martín, Claudia, Rodríguez-Pinzón, Diego y Guevara, José Antonio, Derecho Internacional de los Derechos Humanos, México, Universidad Iberoamericana - Washington College of Law - Fontamara, 2004.

NeUMEYER, ERIK, "Do international human rights treaties improve respect for human rights?", Journal of Conflict Resolution, vol. 49, núm. 6, 2005.

Open Society InItiative, From Judgement to Justice: Implementing International and Regional Human Rights Decisions, Nueva York, Open Society Foundations, 2010.

"Ratification Status Finder”, Oficina del Alto Comisionado de las Naciones Unidas para los Derechos Humanos. [Consulta: 17 de febrero, 2017]. Disponible en: http://tbinternet.ohchr.org/_layouts/TreatyBodyExternal/Treaty.aspx?CountryID=7\&Lang=EN

Risse, Thomas, Ropp, Stephen y Sikkink, Kathryn, The Persistent Power of Human Ri- 
ghts. From Commitment to Compliance, Cambridge, Cambridge University Press, Cambridge, 2013.

Sepúlveda, Magdalena, et al., Human Rights Reference Handbook, San José, Costa Rica, Universidad de la Paz, 2004.

Simmons, Beth, Mobilizing for Human Rights. International Law in Domestic Politics, Cambgridge, Cambridge University Press, 2009.

"Status of Ratifications: Interactive Dashboard", Oficina del Alto Comisionado de las Naciones Unidas para los Derechos Humanos. [Consulta: 17 de febrero, 2017]. Disponible en: http://indicators.ohchr.org/

Steiner, Henry J., Alston, Philip y Goodman, Ryan, International Human Rights in Context. Law, Politics, Morals, Oxford, Oxford University Press, 2008. 